\title{
Staphylococcus aureus protein A binding to osteoblast tumour necrosis factor receptor 1 results in activation of nuclear factor kappa $B$ and release of interleukin- 6 in bone infection
}

\author{
Tânia Claro, ${ }^{1}$ Amro Widaa, ${ }^{1,3}$ Cormac McDonnell, ${ }^{1}$ Timothy J. Foster, ${ }^{2}$ \\ Fergal J. O'Brien ${ }^{3,4}$ and Steven W. Kerrigan ${ }^{1,5}$ \\ ${ }^{1}$ Microbial Infection Group, Molecular and Cellular Therapeutics, Royal College of Surgeons in \\ Ireland, 123 St Stephens Green, Dublin 2, Ireland \\ ${ }^{2}$ Department of Microbiology, Moyne Institute of Preventive Medicine, Trinity College Dublin, \\ Dublin 2, Ireland \\ ${ }^{3}$ Department of Anatomy, Royal College of Surgeons in Ireland, 123 St Stephens Green, Dublin 2, \\ Ireland \\ ${ }^{4}$ Trinity Centre for Bioengineering, Trinity College Dublin, Dublin 2, Ireland \\ ${ }^{5}$ School of Pharmacy, Royal College of Surgeons in Ireland, 123 St Stephens Green, Dublin 2, \\ Ireland
}

Correspondence

Steven W. Kerrigan

skerrigan@rcsi.ie

Received 21 August 2012

Revised 28 September 2012

Accepted 8 November 2012
Staphylococcus aureus is the major pathogen among the staphylococci and the most common cause of bone infections. These infections are mainly characterized by bone destruction and inflammation, and are often debilitating and very difficult to treat. Previously we demonstrated that $S$. aureus protein $\mathrm{A}(\mathrm{SpA})$ can bind to osteoblasts, which results in inhibition of osteoblast proliferation and mineralization, apoptosis, and activation of osteoclasts. In this study we used small interfering RNA (siRNA) to demonstrate that osteoblast tumour necrosis factor receptor-1 (TNFR-1) is responsible for the recognition of and binding to SpA. TNFR-1 binding to SpA results in the activation of nuclear factor kappa $\mathrm{B}(\mathrm{NF} \kappa \mathrm{B})$. In turn, $\mathrm{NF} \kappa \mathrm{B}$ translocates to the nucleus of the osteoblast, which leads to release of interleukin 6 (IL-6). Silencing TNFR-1 in osteoblasts or disruption of the spa gene in $S$. aureus prevented both NF $\kappa$ B activation and IL-6 release. As well as playing a key role in proinflammatory reactions, IL-6 is also an important osteotropic factor. Release of IL-6 from osteoblasts results in the activation of the bone-resorbing cells, the osteoclasts. Consistent with our results described above, both silencing TNFR-1 in osteoblasts and disruption of spa in $S$. aureus prevented osteoclast activation. These studies are the first to demonstrate the importance of the TNFR-1-SpA interaction in bone infection, and may help explain the mechanism through which osteoclasts become overactivated, leading to bone destruction. Anti-inflammatory drug therapy could be used either alone or in conjunction with antibiotics to treat osteomyelitis or for prophylaxis in high-risk patients.

\section{INTRODUCTION}

The skeletal system plays a critical role in the provision of structural support and protection of inner organs of the body (Clarke, 2008). Bone is the major tissue component of the skeletal system, which is constantly undergoing remodelling. The bone remodelling process is a tightly regulated process that is controlled by bone-forming cells,

Abbreviations: FnBP, fibronectin-binding protein; IL-6, interleukin 6; $\mathrm{NF} \kappa \mathrm{B}$, nuclear factor kappa B; siRNA, small interfering RNA; TNF, tumour necrosis factor; TNFR-1, tumour necrosis factor receptor 1; TRAP, tartate-resistant acid phosphatase. osteoblasts and bone-degrading cells, osteoclasts (Eriksen, 2010). Remodelling begins with the laying down of an organic matrix and its calcification by osteoblasts, followed by the removal of this calcified matrix by the osteoclast (Sims \& Gooi, 2008). Bone remodelling is a constant process that enables the skeletal system to respond and adapt to mechanical stresses encountered in everyday life (Hill, 1998). An imbalance in bone remodelling can interfere with osteoblast function and osteoclast activity, leading to the failure of the resorbed bone to be filled by new calcified matrix. As a result, this imbalance causes increased bone fragility, which leads to a high fracture risk 
in affected individuals (Clarke, 2008). There are many factors that can trigger an imbalance, such as disorders of the endocrine system (Lombardi et al., 2011; Karsenty, 2012), but the most sustained and devastating is the presence of an infecting micro-organism in bone (Lew \& Waldvogel, 2004).

Osteomyelitis is an acute or chronic infection of the bone and is characterized by suppurative inflammation, abnormal bone remodelling, together with uncontrolled bone resorption (Montanaro et al., 2011). Micro-organisms can gain entry to the bone using a number of different routes. Contiguous infection occurs following trauma, bone surgery or joint replacement. Haematogenous infection occurs as a result of a bloodstream infection or bacteraemia (Wright \& Nair, 2010). Osteomyelitis can be caused by a wide range of different micro-organisms. However Staphylococcus aureus infection is the most common, accounting for up to $80 \%$ of clinical cases (Berendt \& Byren, 2004). Treatment for osteomyelitis is often unsuccessful and requires radial debridement of the affected limb coupled with intense combination antibiotic treatment (Rao et al., 2011). Of particular concern is the rapid emergence of multiple resistant strains of $S$. aureus and the recent identification of $S$. aureus isolates that are resistant to or have reduced susceptibility to vancomycin, the last remaining antibiotic to which the bacteria was uniformly susceptible (Vander Have et al., 2009).

To date, the mechanism by which $S$. aureus causes weakening of the bones in osteomyelitis is not fully understood. However, significant advances in our understanding of the underlying mechanisms are beginning to emerge. Earlier reports demonstrate that osteoblasts are capable of internalizing $S$. aureus. Uptake is promoted by fibronectin-binding proteins (FnBPs) expressed on $S$. aureus that capture fibronectin and use it as a bridge between bacteria and the $\alpha 5 \beta 1$ integrin expressed on osteoblasts (Sinha et al., 1999; Ahmed et al., 2001). Integrin clustering in the osteoblast results in signalling that leads to bacterial uptake, thus rendering the bacteria safe from both immune and antibiotic attack. Once internalized, S. aureus cells also have the ability to induce apoptosis via the TRAIL/caspase pathway, thus contributing to bone destruction (Tucker et al., 2000). More recently, our group has demonstrated that in the absence of FnBPs, S. aureus is still capable of interacting with osteoblasts (Claro et al., 2011). We demonstrated that $S$. aureus is capable of triggering a series of downstream events that contribute to abnormal bone remodelling. When $S$. aureus protein A binds to osteoblasts, it leads to an increase in the expression of RANKL, an important cytokine involved in osteoclast differentiation. The binding also generates signals in the osteoblast that lead to the inhibition of mineralization and osteoblast proliferation. Finally, we demonstrated that $\mathrm{SpA}$ binding to osteoblasts generates a signal that induces osteoblast apoptosis. Deletion of SpA from $S$. aureus prevented these events from occurring, suggesting that the $S$. aureus $\mathrm{SpA}$-osteoblast interaction is a critical event in upsetting normal bone remodelling (Claro et al., 2011; Widaa et al., 2012).

SpA is a multifunctional protein, which has been previously shown to bind a number of ligands, including tumour necrosis factor receptor 1 (TNFR-1) (Gómez et al., 2006). Osteoblasts express high levels of TNFR-1. In the current study we demonstrate that the TNFR-1 receptor plays a critical role in binding $S$. aureus $\mathrm{SpA}$, and furthermore is responsible for producing interleukin 6 (IL-6) via the nuclear factor kappa B (NF $\kappa \mathrm{B})$ pathway, resulting in osteoclast activation.

\section{METHODS}

Bacterial strains and growth conditions. The bacterial strains used in this study are listed in Table 1. S. aureus strains were grown to stationary phase at $37{ }^{\circ} \mathrm{C}$ in Brain Heart Infusion broth (Oxoid). $S$. aureus strain Newman ( $\mathrm{pCU}_{1} \mathrm{spa}^{+}$) was grown with the addition of $10 \mu \mathrm{g}$ chloramphenicol $\mathrm{ml}^{-1}$ (Sigma-Aldrich). Bacteria were harvested and washed by centrifugation at $4000 \mathrm{~g}$ for $5 \mathrm{~min}$, resuspended in PBS, pH 7.4, and adjusted to $1 \times 10^{9}$ cells $\mathrm{ml}^{-1}$ for all studies.

Tissue cell culture conditions. The mouse clonal MC3T3-E1 preosteoblastic cell line (ATCC) was used for all experiments. This is a common cell line used routinely for investigating osteoblast function. The cells were cultured in standard T175 tissue culture flasks (Sarstedt) containing $\alpha$-MEM supplemented with $10 \%$ FBS (Biosera), $2 \%$ penicillin/streptomycin solution and $1 \%$ L-glutamine (SigmaAldrich). The medium was replaced every 3-4 days, and after confluency, cells were harvested using trypsin-EDTA (SigmaAldrich) and resuspended in the standard medium. MC3T3-E1 preosteoblastic cells were differentiated to matrix-secreting mature osteoblasts using the standard osteogenic cocktail, by supplementation with dexamethasone $(100 \mathrm{nM})$, ascorbic acid $\left(50 \mu \mathrm{g} \mathrm{ml}^{-1}\right)$ and $\beta$-glycercolpentahydrate (10 mM) (Sigma-Aldrich).

The murine RAW 264.7 pre-osteoclast cell line (ATCC) was used for all osteoclast studies. RAW 264.7 cells were cultured in T175 tissue culture flasks in high-glucose Dulbecco's modified Eagle's medium (DMEM) (Sigma-Aldrich) supplemented with $10 \%$ heat inactivated fetal bovine serum (Biosera) and $1 \%$ penicillin/streptomycin solution (Sigma-Aldrich).

Silencing of TNFR-1 on osteoblastic cells using small interfering RNA (siRNA). siRNA silencing was used to knock down TNFR-1 using the murine Amaxa Cell Line Nucleofector kit R (Lonza). Osteoblastic cells $\left(1 \times 10^{9}\right.$ cells $\left.\mathrm{ml}^{-1}\right)$ were harvested and centrifuged at $9000 \mathrm{~g}$ for $5 \mathrm{~min}$ at room temperature. After centrifugation, the supernatant was removed and each pellet was resuspended in $100 \mu \mathrm{l}$ RT Nucleofector Solution R with $150 \mathrm{nM}$ siRNA (Ambion). The suspended cells with siRNA were then electroporated using a Nucleofector I electroporator (ISIS). Immediately after electroporation, $500 \mu \mathrm{l}$ pre-equilibrated osteoblast medium was added to the cells. Following 5 days of growth, osteoblasts were lysed and loss of expression of TNFR-1 was confirmed by Western immunoblotting.

Western immunoblotting. Infected, uninfected and siRNA-treated osteoblasts were lysed in RIPA buffer containing $1 \times$ protease inhibitor cocktail supplemented with phosphatase inhibitors (sodium fluoride, sodium orthovanadate and calyculin A) on ice for $10 \mathrm{~min}$. Cleared lysates were separated on a $10 \%$ SDS-PAGE gel. Proteins were electroblotted onto PVDF membranes (Roche) for $1 \mathrm{~h}$. Membranes were probed with a primary anti-rabbit IgG against TNFR-1 (Santa Cruz), anti-mouse IgG against IkB (Calbiochem) or 
Table 1. S. aureus strains used in this study

\begin{tabular}{|lll|}
\hline Strain & \multicolumn{1}{c|}{ Relevant characteristics } & Reference \\
\hline Newman & NCTC 8178 wild-type. Isolated from a case of secondarily infected & Duthie \& Lorenz (1952) \\
& tubercular osteomyelitis in man & McDevitt et al. (1994) \\
DU5876 Newman clfA & clfA2::Tn917. Defective in clumping factor A & O'Brien et al. (2002) \\
DU5971 Newman spa & spa:: Ka ${ }^{\mathrm{r}}$. Defective in protein A & Claro et al. (2011) \\
Newman pCU1spa+ & $\mathrm{Cm}^{\mathrm{r}}$ Ap $^{\mathrm{r}}$ spa gene cloned into pCU1. Insertion of pCU1spa, capable of & \\
& replicating in Escherichia coli and S. aureus expressing protein A & \\
& &
\end{tabular}

anti-rabbit IgG against $\alpha$-actinin (Santa Cruz) overnight at $4{ }^{\circ} \mathrm{C}$ with constant inversion. Unbound antibody was removed by three $10 \mathrm{~min}$ washes with Tris-buffered saline (TBS) buffer. Protein bands were detected using a species-specific horseradish peroxidase-conjugated secondary antibody and developed by chemiluminescence.

ELISA IL-6 production in osteoblasts following S. aureus binding. The amount of IL-6 released from non-infected and $S$. aureus-infected osteoblasts over 24 and $48 \mathrm{~h}$ was quantified using the Mouse IL-6 ELISA Ready-SET-Go kit (eBioscience). A 96-well microplate was coated with IL-6 capture antibody and sealed with hydrophobic porous sealing film. The plate was then incubated overnight at $4{ }^{\circ} \mathrm{C}$. Following this, wells were washed by addition of $250 \mu \mathrm{l}$ wash buffer. The plate was inverted and blotted on absorbant paper to remove any residual buffer. Each well was blocked with $200 \mu \mathrm{l} 1 \times$ assay diluent and the plate was incubated at room temperature $\left(21^{\circ} \mathrm{C}\right)$ for $1 \mathrm{~h}$. Standards and samples were prepared by diluting mouse recombinant IL- 6 in $1 \times$ assay diluent. The plate was washed again by adding $250 \mu \mathrm{l}$ Wash Buffer per well. Finally, $100 \mu \mathrm{l}$ of standard or sample was added to the 96-well plate. The plate was sealed using hydroporous sealing film and incubated at room temperature for $2 \mathrm{~h}$. After washing the plate, $100 \mu \mathrm{l}$ of detection antibody was added to each well and incubated for $30 \mathrm{~min}$ at room temperature. After three washes of each well with $250 \mu \mathrm{l}$ Wash Buffer, $100 \mu \mathrm{l}$ substrate solution was added and the plate was incubated for $15 \mathrm{~min}$ at room temperature. Finally $50 \mu \mathrm{l}$ stop solution was added and the $A_{450}$ was read using a Wallac Victor2 microplate reader system (Perkin Elmer).

Tartate-resistant acid phosphatase (TRAP) assay for osteoclastogenesis. To determine the effects of $S$. aureus on the formation and proliferation of pre-osteoclasts, RAW 264.7 cells were seeded $\left(2 \times 10^{4}\right.$ cells per well $)$ in 12-well tissue culture plates. Conditioned 4-day medium from osteoblasts, either exposed or unexposed to $S$. aureus, was then transferred to the pre-osteoclasts, which were cultured for a further 4 days. TRAP was quantified to measure osteoclastogenesis on day 4. TRAP quantification was carried out by removing conditioned media from pre-osteoclasts and washing cells in PBS. Cells were then incubated for $1 \mathrm{~h}$ at $37{ }^{\circ} \mathrm{C}$ in $1 \mathrm{mM}$ citrate solution. After the $1 \mathrm{~h}$ incubation, $20 \mu \mathrm{l}$ of supernatant from the wells was added in triplicate to wells of a 96-well plate containing $50 \mu 1100 \mathrm{mM}$ p-nitrophenol phosphate, $80 \mathrm{mM}$ sodium tartrate, $200 \mathrm{mM}$ sodium citrate and $200 \mathrm{mM}$ sodium chloride (Sigma-Aldrich). After $30 \mathrm{~min}$ incubation at $37^{\circ} \mathrm{C}$, the reaction was stopped using $1 \mathrm{M} \mathrm{NaOH}$ and the $A_{405}$ was measured using a microplate reader (Wallac Victor2).

\section{RESULTS}

\section{Osteoblast TNFR-1 binds to S. aureus SpA}

Previously we demonstrated that $S$. aureus SpA binds to and triggers a series of events that contribute to bone destruction; however, the osteoblast receptor to which SpA binds has not been definitively identified. Protein A has been shown to bind a number of ligands, including TNFR1. Osteoblasts express high levels of TNFR-1. Our previous results demonstrated that blocking osteoblast TNFR-1 with an antibody reduced osteoblast adhesion to $S$. aureus Newman. To confirm the interaction between osteoblast TNFR-1 and $S$. aureus SpA we employed siRNA techniques. Osteoblasts were preincubated with an siRNA specifically targeted at knocking down expression of TNFR-1. Western immunoblot analysis confirmed successful silencing of the TNFR-1 expression on the osteoblasts (Fig. 1a). $\alpha$-Actinin was used as a loading control (Fig. 1a). siRNA-treated osteoblasts displayed significantly less binding to $S$. aureus than the untreated osteoblast control (Fig. 1b, $P<0.01$ ). Interestingly, siRNA-treated osteoblasts bound significantly less to the $S$. aureus strain defective in expression of SpA (Fig. 1b, $P<0.05$ ). This suggests that TNFR-1 bound to an additional adhesin (other than SpA) on S. aureus.

\section{S. aureus SpA binding to TNFR-1 induces NF $\kappa$ B activation}

As well as the TNFR-1 being recognized as a death receptor it also plays an important role in the control of gene transcription through the activation of $\mathrm{NF} \kappa \mathrm{B}$. $\mathrm{NF} \kappa \mathrm{B}$ is a dimeric protein which is translocated from the cytoplasm to the nucleus under the control of the inhibitory protein IkB (Carmody \& Chen 2007), where it activates transcriptional factors that mediate the expression of inflammatory cytokines and chemokines (Panzer et al., 2009). As a measure of $\mathrm{NF} \kappa \mathrm{B}$ activation, degradation of $\operatorname{Ik} \beta$ was investigated by immunoblotting. Osteoblasts were infected over a $90 \mathrm{~min}$ period with $S$. aureus Newman. IkB degraded in a time-dependent manner over a $90 \mathrm{~min}$ period following infection of osteoblasts with $\mathrm{SpA}+S$. aureus Newman (Fig. 2a). Following infection with an SpAdefective mutant, no IkB degradation was detected over the 90 min period (Fig. 2b). The complemented mutant $\left(\mathrm{pCU} 1 \mathrm{spa}^{+}\right.$) recovered the ability to induce degradation of $\mathrm{IkB}$ over the $90 \mathrm{~min}$ period (Fig. 2c). Pre-incubating osteoblasts with an anti-TNFR-1 IgG (Fig. 2d) or using TNFR-1-silenced osteoblasts failed to induce IkB degradation in the presence of $S$. aureus Newman (Fig. 2d, e). These results demonstrate that in the absence of SpA on the $S$. aureus surface or in TNFR-1-blocked or -silenced 
(a)

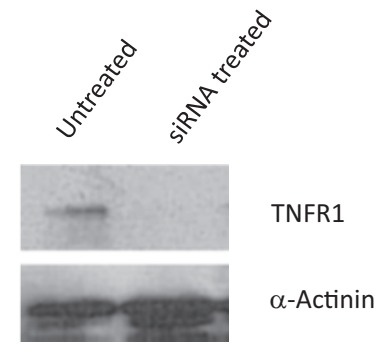

(b)

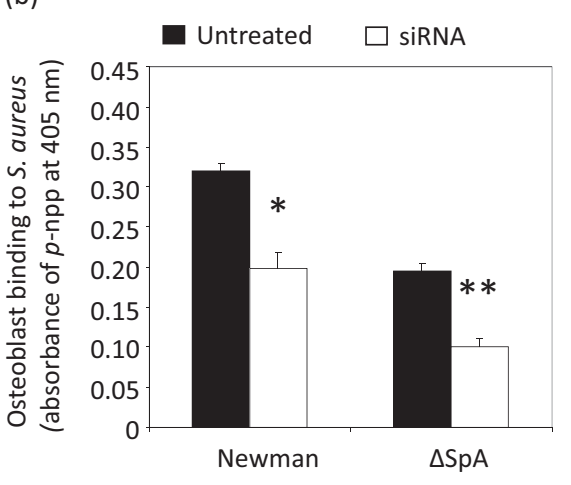

S. aureus strain
Fig. 1. TNFR-1 is the receptor for SpA on the osteoblast surface. (a) siRNA-negative and -positive osteoblast lysates were separated on a $10 \%$ SDS-PAGE gel and electroblotted onto PVDF membranes. Membranes were probed with rabbit polyclonal antiTNFR- 1 and $\alpha$-actinin as an equal loading control. Protein bands were detected using rabbit horseradish peroxidase-conjugated secondary antibody and chemiluminescence. (b) siRNA was used to silence TNFR-1 on osteoblasts. Osteoblasts were allowed to adhere to $S$. aureus Newman wild-type for $45 \mathrm{~min}$ at $37{ }^{\circ} \mathrm{C}$. Binding was determined by measuring the intracellular alkaline phosphatase content at $405 \mathrm{~nm}$ in a microplate reader. $p$-npp, $p$ Nitrophenol phosphate. ${ }^{\star} P<0.01,{ }^{\star \star} P<0.05$; error bars, SEM, $n=3$.

osteoblasts, the TNFR-1 pathway is not activated. As a consequence, $\mathrm{IkB}$ retains $\mathrm{NF} \kappa \mathrm{B}$ in the cytosol, preventing the transcription of genes involved in the inflammatory response.

\section{S. aureus SpA binds to osteoblast TNFR-1 and causes release of IL-6}

Recent observations suggest that activated osteoblasts might serve as a source of proinflammatory cytokines. $\mathrm{NF} \kappa \mathrm{B}$ activates transcriptional factors that mediate the expression of several proinflammatory cytokines. IL-6 is an example of one such proinflammatory cytokine that is found at high levels in patients with bone infection. We next investigated whether $S$. aureus SpA binding to osteoblast TNFR-1 leads to activation of $\mathrm{NF} \kappa \mathrm{B}$ and thus secretion of IL-6 into the local environment. S. aureus strains were preincubated with untreated or siRNA-treated (a)

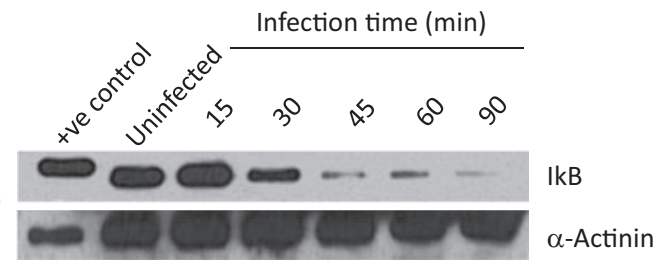

(b)

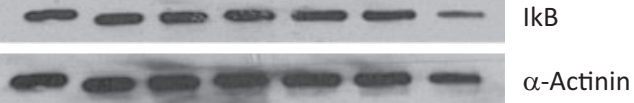

(c)

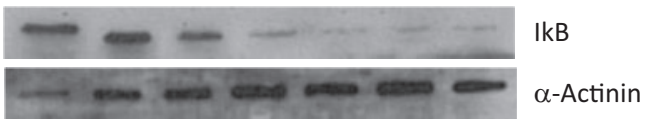

(d)

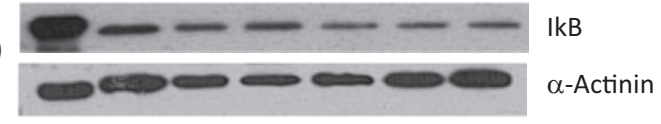

(e)

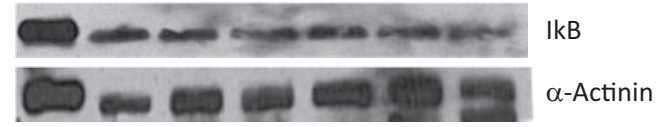

Fig. 2. SpA binding to TNFR-1 induces $N F \kappa B$ activation. IkB expression over a 90 min period in normal osteoblasts following infection with (a) S. aureus Newman wild-type, (b) $\Delta \mathrm{SpA}$, (c) pCu1SpA, (d) anti-TNFR-1 antibody and (e) TNFR-1-silenced osteoblasts. Uninfected and infected osteoblast lysates were separated on a $10 \%$ SDS-PAGE gel and electroblotted onto PVDF membranes. Membranes were probed with monoclonal lkB antibody. Protein bands were detected using a mouse horseradish peroxidase-conjugated secondary antibody and chemiluminescence. Immunoblots are representative of three independent experiments.

osteoblasts for $48 \mathrm{~h}$ before the supernantent was collected and assayed for IL-6. Uninfected osteoblasts released low levels of IL-6 compared with those incubated with $S$. aureus Newman, which caused an almost eightfold increase of IL6 (Fig. 3a, $P<0.001$ ). In contrast, the SpA-defective mutant significantly reduced IL-6 release (Fig. 3a, $P<0.001$, compared with the wild-type). The complemented mutant $\left(\mathrm{pCU} 1 s p a^{+}\right.$) stimulated a similar level of IL-6 to that of wild-type $S$. aureus Newman (Fig. 3a, $P$ value not significant).

To further investigate whether $S$. aureus SpA binding to osteoblast TNFR-1 caused the release of IL-6 we used osteoblasts pretreated with siRNA to knock down expression of TNFR-1. Consistent with our earlier observations, uninfected untreated osteoblasts failed to release IL-6. In contrast to those preincubated with siRNA, untreated osteoblasts with $S$. aureus showed a significant increase in IL-6 release (Fig. 3b, $P<0.0001$ ). The siRNA-treated osteoblasts did not release IL-6 when challenged with $S$. aureus (Fig. 3b, $P<0.0001$ ). These results suggest that $S$. aureus $\mathrm{SpA}$ binds to osteoblast TNFR-1, causing the release of the proinflammatory cytokine IL- 6 . 
(a)

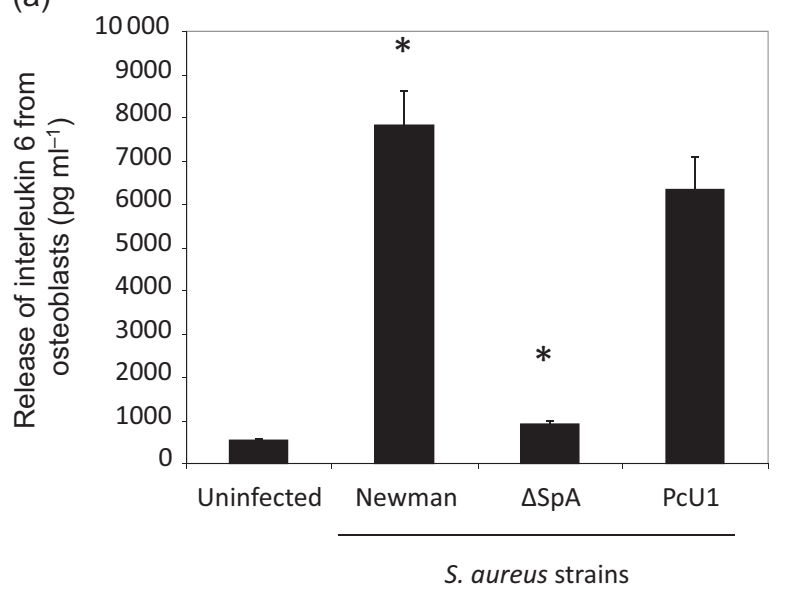

(b)

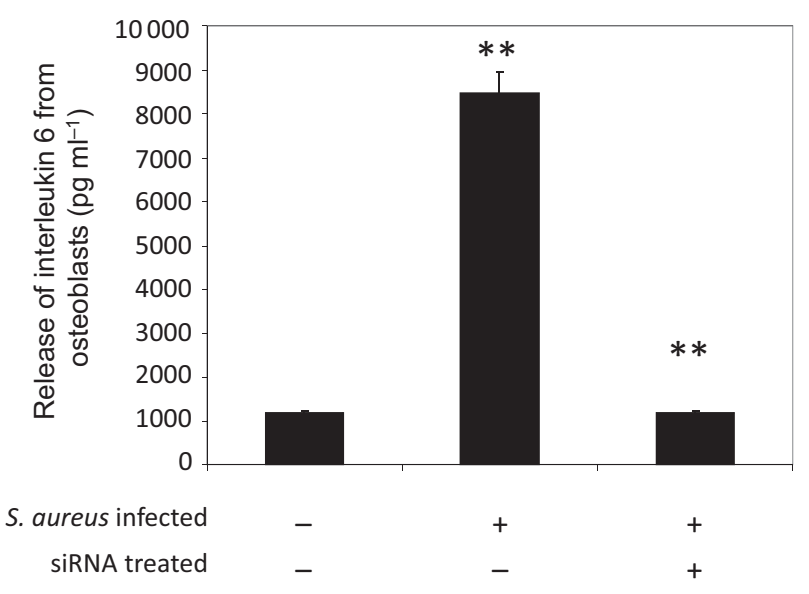

Fig. 3. SpA binding to TNFR-1 induces IL-6 release on osteoblasts. (a) The amount of IL-6 released from non-infected and $S$. aureus-infected osteoblasts over 24 and $48 \mathrm{~h}$ was quantified using the mouse IL-6 ELISA. (b) The levels of IL-6 released were quantified in supernatants of both uninfected and $S$. aureus-infected normal and TNFR-1-silenced osteoblasts, over $48 \mathrm{~h}$ infection times. ${ }^{\star} P<0.001,{ }^{\star *} P<0.0001$; error bars, SEM, $n=3$.

\section{S. aureus causes activation of osteoclasts}

Having demonstrated the release of IL-6 from osteoblasts as a result of SpA binding to TNFR-1, we next determined whether the released IL-6 triggers osteoclastogenesis. To address this, medium was removed from both uninfected and S. aureus-infected osteoblasts and transferred to preosteoclasts for up to 21 days. Media from S. aureus-infected osteoblasts contains many secreted factors, including IL-6. TRAP is a glycosylated monomeric metalloenzyme that is highly expressed in osteoclasts, and its detection is utilized as an indicator of osteoclastogenesis. TRAP levels were quantified from osteoclastic cells after 4 days culture in media taken from uninfected and $S$. aureus-infected osteoblastic cells. TRAP activity from osteoclastic cells cultured in S. aureus Newman wild-type infected medium was higher than than that from cells cultured in the uninfected osteoblastic cell culture medium (Fig. 4, $P<0.01)$. The SpA mutant failed to induce higher TRAP levels than the uninfected cells (Fig. $4, P$ value not significant), while the complemented mutant ( $\left.\mathrm{pcU} 1 s p a^{+}\right)$ produced a higher level of TRAP (Fig. $4, P<0.001$ ), at a similar levels to wild-type $S$. aureus Newman (Fig. 4, $P$ value not significant).

We next addressed the role of osteoblast TNFR-1. siRNA silencing of TNFR-1 on the osteoblastic cells failed to increase the TRAP level compared with uninfected osteoblasts when cultured in the presence of $S$. aureus Newman wild-type strain (Fig. $4, P$ value not significant). These results further confirm that $S$. aureus $S p A$ binds to osteoblast TNFR-1, inducing the release of IL-6, which leads to activation of osteoclasts.

\section{DISCUSSION}

S. aureus is a commensal of humans found predominantly in the moist squamous epithelium of the anterior nares (Foster, 2009). It is also an important pathogen, causing many types of infection, including osteomyelitis. Trauma, surgery, joint replacement or bloodstream infection provides routes of entry for these bacteria into the bone tissue (Lew \& Waldvogel, 2004). Osteomyelitis is characterized by progressive inflammatory destruction of the bone (Wright \& Nair, 2010). The ability of S. aureus to bind to and trigger intracellular signals in the osteoblast is considered to be a primary event in the pathogenesis of osteomyelitis.

Understanding the mechanisms through which $S$. aureus binds to osteoblasts and generates intracellular signals leading to bone loss and bone destruction in vitro is poorly characterized. Recently, a model of the early stages of infection was proposed, where $S$. aureus FnBPs bind fibronectin, which in turn bridges to osteoblast $\alpha 5 \beta 1$, facilitating internalization (Sinha et al., 1999; Ahmed et al., 2001). Using this strategy, S. aureus can evade immune cell recognition or antibiotic attack by hiding inside osteoblasts. SpA can promote binding to osteoblasts, which results in activation of osteoclasts and inhibition of osteoblast proliferation and mineralization. In addition, SpA binding to osteoblasts induces apoptosis via caspase 6 activation (Claro et al., 2011; Widaa et al., 2012). These effects are believed to contribute to the bone loss observed in osteomyeltitis patients.

In the present study we demonstrate that $S$. aureus $\mathrm{SpA}$ binds to TNFR-1 on osteoblasts. A key target involved in the downstream signalling cascade of TNFR-1 is IkB degradation and $\mathrm{NF} \kappa \mathrm{B}$ activation (Guicciardi \& Gores, 2009). Disruption of the spa gene on S. aureus or silencing expression of TNFR-1 on osteoblasts prevented IkB degradation and $\mathrm{NF} \kappa \mathrm{B}$ activation. IL-6 is an important 


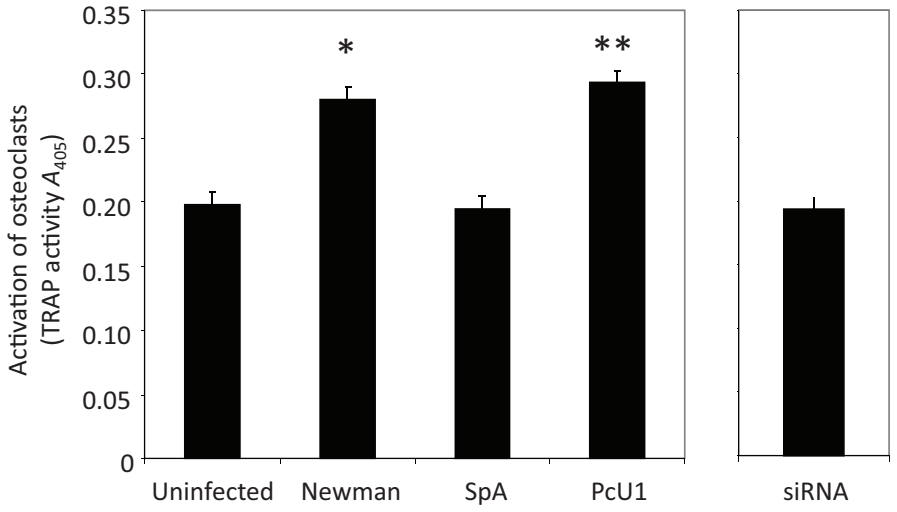

Fig. 4. S. aureus causes activation of osteoclasts. Pre-osteoclasts were seeded $\left(2 \times 10^{4}\right.$ cells per well) in 12-well tissue culture plates and cultured for 21 days. Conditioned media from osteoblasts (4 days), with either control buffer or formaldehyde-fixed $S$. aureus Newman, Newman $\Delta$ spa or Newman spa (pCU1spa+), was then transferred to the pre-osteoclasts. Osteoclastogenesis was determined by measuring TRAP. ${ }^{\star} P<0.01,{ }^{\star \star} P<$ $0.001, n=3-5$. The right-hand panel shows TRAP activity after siRNA silencing of TNFR-1. proinflammatory cytokine that is produced as a result of $\mathrm{NF} \kappa \mathrm{B}$ activation. IL-6 also plays a key role in activating the bone-resorbing cells, the osteoclasts (Ishimi et al., 1990). Upon $\mathrm{NF} \kappa \mathrm{B}$ activation, osteoblasts release IL-6, which subsequently activates osteoclasts. Neither the SpA mutant nor the TNFR-1-silenced osteoblast was able to release IL-6 and trigger activation of osteoclasts. This is a particularly important step in the development of osteomyelitis because this pathway may account for the increased bone resorption and subsequent bone loss experienced in osteomyelitis patients.

Significant emphasis has been placed on the role of TNFR1 in cell death, and it is now generally known as a death receptor. However, more recently, TNFR-1 has also been shown to play a key role in cytokine generation and inflammation. For example, $S$. aureus $\mathrm{SpA}$ was previously shown to bind TNFR-1 in lung epithelial cells, resulting in proinflammatory signalling in the pathogenesis of staphylococcal pneumonia (Gómez et al., 2006). In the current study we demonstrate that osteoblasts express TNFR-1 and that silencing TNFR-1 on osteoblasts significantly reduces binding to $S$. aureus. Interestingly, binding of the SpA mutant to TNFR-1-silenced osteoblasts was also significantly reduced, suggesting that SpA may also be binding to another protein on the osteoblast surface. One distinct possibility is epidermal growth factor receptor (EGFR), as this is also expressed on osteoblasts (data not shown) and is a ligand for SpA (Gómez et al., 2007).

A key signalling component downstream of TNFR-1 is $\mathrm{NF} \kappa \mathrm{B}$. NF $\kappa \mathrm{B}$ is a dimeric protein composed of members of the Rel/NF $\kappa \mathrm{B}$ family. Typically, $\mathrm{NF} \kappa \mathrm{B}$ dimers are retained in the cytoplasm by inhibitory protein $\operatorname{IkB} \alpha$ (Kriete \& Mayo, 2009). Upon phosphorylation, $\operatorname{IkB} \alpha$ becomes ubiquitinated and degraded which finally causes activation of NF $\kappa \mathrm{B}$ (Carmody \& Chen, 2007). A number of bacteria have been shown to activate $\mathrm{NF} \kappa \mathrm{B}$ in response to infection in various cell types, including Streptococcus pneumoniae in lung epithelial cells (Scharf et al., 2012), Streptococcus pyogenes in macrophages (Hertzén et al., 2010) and S. aureus in endothelial cells (Oviedo-Boyso et al., 2011). Here we demonstrate that $S$. aureus SpA binding to osteoblast TNFR-1 induces activation of NF $\kappa$ B. Either deleting $\mathrm{SpA}$ from $S$. aureus or reducing TNFR-1 expression by osteoblasts prevented activation of $\mathrm{NF} \kappa \mathrm{B}$. This is an important observation, as $\mathrm{NF} \kappa \mathrm{B}$ is a critical molecular switch for several downstream events that affect host responses to infection and immune regulation. For example, once activated, free $\mathrm{NF} \kappa \mathrm{B}$ translocates into the nucleus of the cell to initiate the transcription of many $\mathrm{NF} \kappa \mathrm{B}$-dependent genes, which include growth factors, adhesion molecules, chemokines and cytokines (Ning et al., 2010). Using a mouse model of osteomyelitis there was a significant increase in cytokines IL- $1 \beta, \mathrm{TNF} \alpha$ and IL-6 following infection with S. aureus (Yoshii et al., 2002; García-Alvarez et al., 2009). The source of these cytokines was not fully clear as $S$. aureus infection led to increased transcription, but not increased protein synthesis or secretion of IL-1 $\beta$ from murine osteoblasts (Marriott et al., 2002). Furthermore osteoblasts only release low levels of tumour necrosis factor (TNF) $\alpha$ (Bu et al., 2003). IL-6 is a pleiotropic cytokine that acts as a regulator of immune response and inflammation during infection or trauma (Kishimoto et al., 1992). There is growing evidence that IL-6 is an important osteotropic factor. IL-6 is produced by osteoblasts in response to $S$. aureus infection and has been shown to stimulate osteoclastic bone resorption (Ishimi et al., 1990). In the current study and consistent with previous reports, we demonstrated that osteoblasts secrete high levels of IL-6 following binding of S. aureus and that this contributed to osteoclast activation. This was dependent on SpA expression by S. aureus and TNFR-1 expression by the osteoblasts.

Significant progress has been made recently in outlining the mechanisms through which $S$. aureus induces bone destruction and bone loss. Previous reports suggest a dual mechanism to bone infection by $S$. aureus. In the first mechanism, S. aureus FnBPs bind fibronectin and bridge the bacteria to osteoblast $\alpha 5 \beta 1$. This mechanism contributes to immune evasion through internalization into the osteoblast and persistence of infection. In the second mechanism, S. aureus SpA binds osteoblast TNFR-1 and triggers a series of events that lead to inhibition of mineralization, inhibition of osteoblast proliferation and 


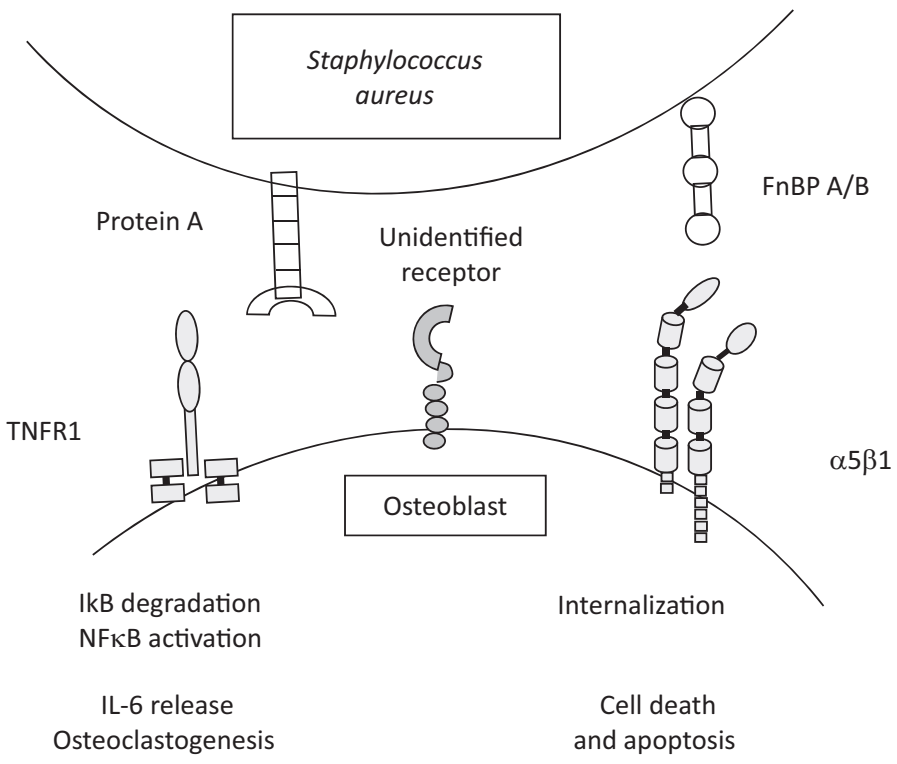

Fig. 5. Model of $S$. aureus interaction with osteoblasts. Engagement of $S$. aureus protein A with osteoblast TNFR-1 results in $\mathrm{kBB}$ degradation and $\mathrm{NF} \kappa \mathrm{B}$ activation. This results in the release of IL-6, which in turn activates the bone-resorbing cells, osteoclasts. A second mechanism exists in which $S$. aureus FnBP A/B binds fibronectin bridges to $\alpha 5 \beta 1$ on the osteoblast and triggers internalization, resulting in cell death and apoptosis.

induction of apoptosis, contributing to bone loss. The current study builds on these observations by demonstrating that SpA binding to TNFR-1 also triggers IL-6 release via activation of $\mathrm{NF} \kappa \mathrm{B}$ (Fig. 5). In turn, IL-6 activates the bone-resorbing cells, osteoclasts, which contributes to bone destruction. The significance of the current work lies in the discovery that IL-6 release from $S$. aureus-infected osteoblasts triggers activation of osteoclasts. In bone disease, IL-6 inhibitors have been used to treat postmenopausal osteoporosis and rheumatoid arthritis (Edwards \& Williams, 2010; Jazayeri et al., 2010), indicating the benefits of preventing IL- 6 responses in the bone. The involvement of osteoblast TNFR-1 in mediating the release of IL-6, which then activates the bone-resorptive cells, osteoclasts, makes the receptor a potential target for therapeutic intervention. This would prevent the destruction and loss of bone in osteomyelitis patients. Novel drug therapies could be used in conjunction with antibiotics to treat osteomyelitis or on their own as a prophylaxis for high-risk patients.

While significant advances are being made in understanding the complex interplay between $S$. aureus and osteoblasts, much work in this area is still needed. For example, deletion of $S$. aureus SpA only reduces osteoblast binding by roughly $70 \%$, suggesting that additional interactions between $S$. aureus and osteoblasts occur. S. aureus expresses many potential candidates, including other LPXTGanchored proteins, peptidoglycan and carbohydrates, which have already been shown to be involved in host recognition. In addition, $S$. aureus also secretes several superantigens and enterotoxins, which may also play a role in either the recognition or activation of osteoblasts, and which cannot be ruled out at this stage with respect to whether they play a role in bone infection. One major limitation of this study and of a lot of the previous investigational studies is that all experiments have been carried out in vitro using cell culture techniques. To establish whether these events occur in vivo, future studies should be carried out using animal models of osteomyelitis.

\section{ACKNOWLEDGEMENTS}

This work was supported by a grant from the Science Foundation of Ireland Research Frontiers Programme awarded to S.W.K. (no. BMT/2008/1709) and by Health Research Board of Ireland project grant $\mathrm{RP} / 2006 / 2$ to T. J. F.

\section{REFERENCES}

Ahmed, S., Meghii, S., Williams, R. J., Henderson, B., Brock, J. H. \& Nair, S. P. (2001). Staphylococcus aureus fibronectin binding proteins are essential for internalization by osteoblasts but do not account for differences in intracellular levels of bacteria. Infect Immun 69, 28722877.

Berendt, T. \& Byren, I. (2004). Bone and joint infection. Clin Med 4, 510-518.

Bu, R., Borysenko, C. W., Li, Y., Cao, L., Sabokbar, A. \& Blair, H. C. (2003). Expression and function of TNF-family proteins and receptors in human osteoblasts. Bone 33, 760-770.

Carmody, R. J. \& Chen, Y. H. (2007). Nuclear factor- $\kappa$ B: activation and regulation during Toll-like receptor signaling. Cell Mol Immunol 4, 31-41.

Clarke, B. (2008). Normal bone anatomy and physiology. Clin J Am Soc Nephrol 3 (Suppl. 3), S131-S139.

Claro, T., Widaa, A., O'Seaghdha, M., Miajlovic, H., Foster, T. J., O'Brien, F. J. \& Kerrigan, S. W. (2011). Staphylococcus aureus protein A binds to osteoblasts and triggers signals that weaken bone in osteomyelitis. PLoS ONE 6, e18748.

Duthie, E. S. \& Lorenz, L. L. (1952). Staphylococcal coagulase; mode of action and antigenicity. J Gen Microbiol 6, 95-107.

Edwards, C. J. \& Williams, E. (2010). The role of interleukin-6 in rheumatoid arthritis-associated osteoporosis. Osteoporos Int 21, 12871293. 
Eriksen, E. F. (2010). Cellular mechanisms of bone remodeling. Rev Endocr Metab Disord 11, 219-227.

Foster, T. J. (2009). Colonization and infection of the human host by staphylococci: adhesion, survival and immune evasion. Vet Dermatol 20, 456-470.

García-Alvarez, F., Navarro-Zorraquino, M., Castro, A., Grasa, J. M., Pastor, C., Monzón, M., Martínez, A., García-Alvarez, I., Castillo, J. \& Lozano, R. (2009). Effect of age on cytokine response in an experimental model of osteomyelitis. Biogerontology 10, 649-658.

Gómez, M. I., O’Seaghdha, M., Magargee, M., Foster, T. J. \& Prince, A. S. (2006). Staphylococcus aureus protein A activates TNFR1 signaling through conserved IgG binding domains. J Biol Chem 281, 20190-20196.

Gómez, M. I., Seaghdha, M. O. \& Prince, A. S. (2007). Staphylococcus aureus protein A activates TACE through EGFR-dependent signaling. EMBO J 26, 701-709.

Guicciardi, M. E. \& Gores, G. J. (2009). Life and death by death receptors. FASEB J 23, 1625-1637.

Hertzén, E., Johansson, L., Wallin, R., Schmidt, H., Kroll, M., Rehn, A. P., Kotb, M., Mörgelin, M. \& Norrby-Teglund, A. (2010). M1 protein-dependent intracellular trafficking promotes persistence and replication of Streptococcus pyogenes in macrophages. J Innate Immun 2, 534-545.

Hill, P. A. (1998). Bone remodelling. Br J Orthod 25, 101-107.

Ishimi, Y., Miyaura, C., Jin, C. H., Akatsu, T., Abe, E., Nakamura, Y., Yamaguchi, A., Yoshiki, S., Matsuda, T. \& other authors (1990). IL-6 is produced by osteoblasts and induces bone resorption. J Immunol 145, 3297-3303.

Jazayeri, J. A., Carroll, G. J. \& Vernallis, A. B. (2010). Interleukin-6 subfamily cytokines and rheumatoid arthritis: role of antagonists. Int Immunopharmacol 10, 1-8.

Karsenty, G. (2012). The mutual dependence between bone and gonads. J Endocrinol 213, 107-114.

Kishimoto, T., Akira, S. \& Taga, T. (1992). Interleukin-6 and its receptor: a paradigm for cytokines. Science 258, 593-597.

Kriete, A. \& Mayo, K. L. (2009). Atypical pathways of NF- $\kappa$ B activation and aging. Exp Gerontol 44, 250-255.

Lew, D. P. \& Waldvogel, F. A. (2004). Osteomyelitis. Lancet 364, 369-379. Lombardi, G., Di Somma, C., Rubino, M., Faggiano, A., Vuolo, L., Guerra, E., Contaldi, P., Savastano, S. \& Colao, A. (2011). The roles of parathyroid hormone in bone remodeling: prospects for novel therapeutics. J Endocrinol Invest 34 (7 Suppl.), 18-22.

Marriott, I., Hughes, F. M., Jr \& Bost, K. L. (2002). Bacterial infection of osteoblasts induces interleukin- $1 \beta$ and interleukin-18 transcription but not protein synthesis. J Interferon Cytokine Res 22, 1049-1055.

McDevitt, D., Francois, P., Vaudaux, P. \& Foster, T. J. (1994). Molecular characterization of the clumping factor (fibrinogen receptor) of Staphylococcus aureus. Mol Microbiol 11, 237-248.

Montanaro, L., Testoni, F., Poggi, A., Visai, L., Speziale, P. \& Arciola, C. R. (2011). Emerging pathogenetic mechanisms of the implantrelated osteomyelitis by Staphylococcus aureus. Int J Artif Organs 34, 781-788.
Ning, R., Zhang, X., Guo, X. \& Li, Q. (2010). Attachment of Staphylococcus aureus is required for activation of nuclear factor kappa B in human osteoblasts. Acta Biochim Biophys Sin (Shanghai) 42, 883-892.

O'Brien, L., Kerrigan, S. W., Kaw, G., Hogan, M., Fitzgerald, D. J., Foster, T. \& Cox, D. (2002). Multiple mechanisms for the activation of human platelet aggregation by Staphylococcus aureus: roles for the clumping factors ClfA and ClfB and the serine-aspartate repeat protein SdrE. Mol Microbiol 44, 1033-1044.

Oviedo-Boyso, J., Cortés-Vieyra, R., Huante-Mendoza, A., Yu, H. B., Valdez-Alarcón, J. J., Bravo-Patiño, A., Cajero-Juárez, M., Finlay, B. B. \& Baizabal-Aguirre, V. M. (2011). The phosphoinositide-3kinase-Akt signaling pathway is important for Staphylococcus aureus internalization by endothelial cells. Infect Immun 79, 4569-4577.

Panzer, U., Steinmetz, O. M., Turner, J.-E., Meyer-Schwesinger, C., von Ruffer, C., Meyer, T. N., Zahner, G., Gómez-Guerrero, C., Schmid, R. M. \& other authors (2009). Resolution of renal inflammation: a new role for NF-kappaB1 (p50) in inflammatory kidney diseases. Am J Physiol Renal Physiol 297, F429-F439.

Rao, N., Ziran, B. H. \& Lipsky, B. A. (2011). Treating osteomyelitis: antibiotics and surgery. Plast Reconstr Surg 127 (Suppl. 1), 177S-187S.

Scharf, S., Zahlten, J., Szymanski, K., Hippenstiel, S., Suttorp, N. \& N'Guessan, P. D. (2012). Streptococcus pneumoniae induces human $\beta$ defensin-2 and -3 in human lung epithelium. Exp Lung Res 38, 100110.

Sims, N. A. \& Gooi, J. H. (2008). Bone remodeling: multiple cellular interactions required for coupling of bone formation and resorption. Semin Cell Dev Biol 19, 444-451.

Sinha, B., François, P. P., Nüsse, O., Foti, M., Hartford, O. M., Vaudaux, P., Foster, T. J., Lew, D. P., Herrmann, M. \& Krause, K. H. (1999). Fibronectin-binding protein acts as Staphylococcus aureus invasin via fibronectin bridging to integrin $\alpha_{5} \beta_{1}$. Cell Microbiol 1, 101-117.

Tucker, K. A., Reilly, S. S., Leslie, C. S. \& Hudson, M. C. (2000). Intracellular Staphylococcus aureus induces apoptosis in mouse osteoblasts. FEMS Microbiol Lett 186, 151-156.

Vander Have, K. L., Karmazyn, B., Verma, M., Caird, M. S., Hensinger, R. N., Farley, F. A. \& Lubicky, J. P. (2009). Community-associated methicillin-resistant Staphylococcus aureus in acute musculoskeletal infection in children: a game changer. J Pediatr Orthop 29, 927-931.

Widaa, A., Claro, T., Foster, T. J., O'Brien, F. J. \& Kerrigan, S. W. (2012). Staphylococcus aureus protein A plays a critical role in mediating bone destruction and bone loss in osteomyelitis. PLoS ONE 7, e40586.

Wright, J. A. \& Nair, S. P. (2010). Interaction of staphylococci with bone. Int J Med Microbiol 300, 193-204.

Yoshii, T., Magara, S., Miyai, D., Nishimura, H., Kuroki, E., Furudoi, S., Komori, T. \& Ohbayashi, C. (2002). Local levels of interleukin-1 $\beta,-4$, -6 and tumor necrosis factor $\alpha$ in an experimental model of murine osteomyelitis due to Staphylococcus aureus. Cytokine 19, 59-65.

Edited by: H. Ingmer 